\title{
Double Para-testicular Cellular Angiofibroma and Synchronous Testicular Microlithiasis
}

\author{
Seungkoo Lee $\cdot$ Hyuck Jae Choi $^{1}$ \\ Departments of Anatomic Pathology and ${ }^{1}$ Radiology, Kangwon National University Hospital, Chuncheon, Korea
}

Cellular angiofibroma is a rare benign mesenchymal tumor. Since first described by Nucci et al. in 1997, ${ }^{1}$ this distinct histopathologic entity has been reported in a series of studies ${ }^{2-4}$ and case reports. ${ }^{5-7}$ The tumor is characterized by two principal components, cellular spindle cells and prominent blood vessels. ${ }^{1}$ Mild cytologic atypia and a few mitotic figures have been demonstrated in some cases. ${ }^{2,4}$ The tumor occurs mainly in the inguinoscrotal region in men. ${ }^{2-5,7}$ The molecular pathogenesis of cellular angiofibroma is largely unknown, although retinoblastoma 1 (RB1) and forkhead box protein O1 (FOXO1) have been implicated in some cases. ${ }^{3,7}$ Testicular microlithiasis is a relatively rare condition characterized by calcifications within the seminiferous tubules. Two different types of testicular microlithiasis, hematoxylin bodies and laminated calcifications, have been described. ${ }^{8}$ Many laminated calcifications have been associated with atrophic seminiferous tubules or germ cell neoplasia. ${ }^{8,9}$ Here, we present a case of double para-testicular cellular angiofibroma and synchronous testicular microlithiasis.

\section{CASE REPORT}

The present study was approved by the Institutional Review Board of Kangwon National University Hospital (KNUH-201505-011). A 40-year-old man with two children presented with complaints of self-limited hemospermia for one month. There

\footnotetext{
Corresponding Author

Seungkoo Lee, MD

Department of Anatomic Pathology, Kangwon National University Hospital, Kangwon National University School of Medicine, 156 Baengnyeong-ro, Chuncheon 24289,

Korea

Tel: +82-33-258-9172, Fax: +82-33-258-2475, E-mail: jsklee@kangwon.ac.kr
}

Received: May 11, 2015 Revised: June 22, 2015

Accepted: June 23, 2015 was no history of exposure to sexually transmitted diseases or penile trauma. He had no previous episodes of hematuria. A solid mass-like lesion was first discovered in the left scrotum by selfexamination. Physical examination revealed a non-tender, firm, slightly mobile, and well-demarcated scrotal mass. Ultrasound examination revealed an approximately $1.2-\mathrm{cm}$ solid mass in the tail of the epididymis (Fig. 1A). The mass showed an isoechoic and mild hypervascular pattern. However, the left testis revealed no mass lesion. Prostate examination, urinalysis, and other laboratory studies were unremarkable. The patient underwent a left simple orchiectomy. On gross examination, two para-testicular masses were identified (Fig. 1B). One mass $(1.2 \times 0.8 \times 0.7$ $\mathrm{cm}$ ) was present near the tail of epididymis. The other mass (1.1 $\times 0.6 \times 0.5 \mathrm{~cm})$ was located near the head of epididymis. The two masses were well-circumscribed, and $1.3 \mathrm{~cm}$ and $0.5 \mathrm{~cm}$ apart from the testis, respectively. The cut surface of the mass was grayish white and firm. Microscopically, the two masses showed histologically identical features, which were loosely arranged, bland spindle cells in a myxoid to collagenous stroma and prominent small- to medium-sized blood vessels (Fig. 2A). Two mitotic figures per 50 high power fields were identified only in the large mass (Fig. 2B). A few atypical spindle cells were also present (Fig. 2C). Immunohistochemically, tumor cells of the two masses were mostly positive for vimentin (1:200, Lab Vision, Fremont, CA, USA). Most perivascular spindle cells and some stromal spindle cells were positive for smooth muscle actin (Bond RTU Ab, alpha sm-1, Leica Biosystems, Newcastle upon Tyne, UK). Staining for CD34 (1:200, Lab Vision) was negative in the stromal spindle cells but positive in the perivascular spindle cells and endothelial cells. Tumor cells were negative for S-100 protein (1:100, Lab Vision), desmin (1:100, Zymed, San Francisco, CA, USA), and pan-cytokeratin (1:100, Leica). A moderate num- 

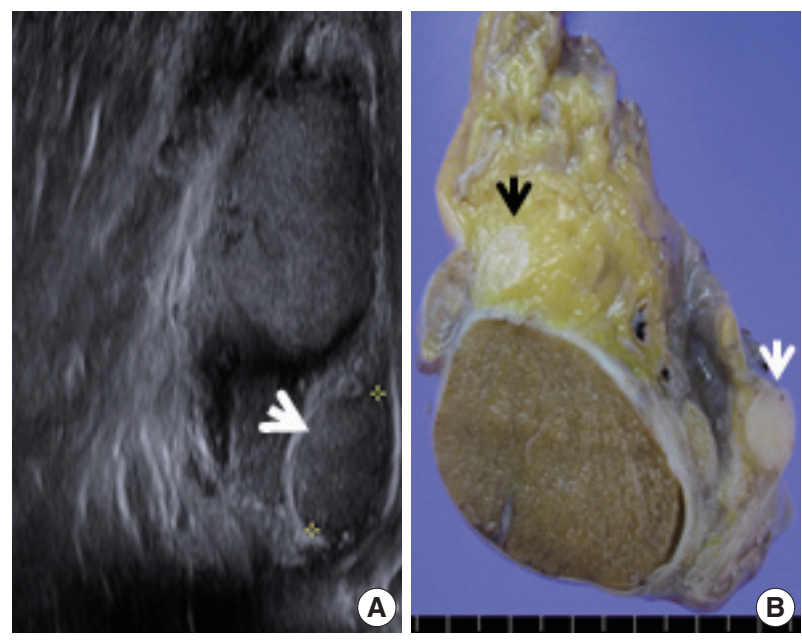

Fig. 1. Ultrasound examination and gross evaluation of orchiectomy specimen. (A) On ultrasound examination, there is an isoechoic mass in the tail area of the epididymis (white arrow). On the cut surface of the gross specimen, solid soft tissue is noted near the tail of the epididymis ( $A$, white arrow) and near the head of the epididymis (B, black arrows).
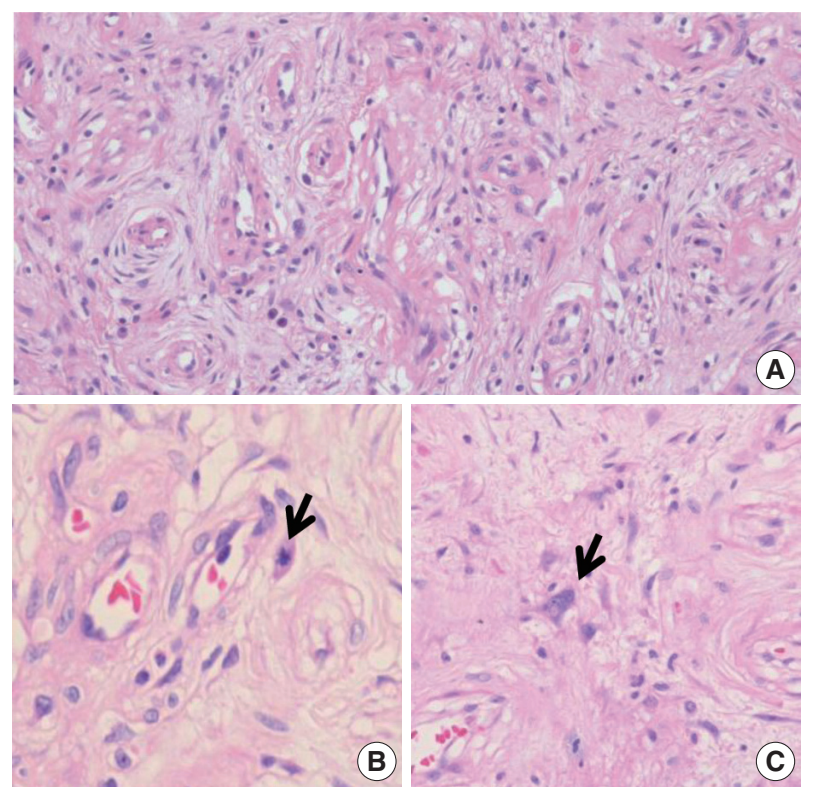

Fig. 2. Histopathologic features of the tumor. (A) The tumor is composed of spindle-shaped mesenchymal cells and thick-walled blood vessels. (B) Two mitotic figures per 50 high-power fields are identified in the mass. There is one of the mitotic cells (arrow). (C) A few stromal cells show atypical nuclear morphology.

ber of lymphocytes and plasma cells were scattered diffusely throughout the stroma of the tumor. Most of these lymphocytes were $\mathrm{CD} 8+$ cytotoxic $\mathrm{T}$ cells. The two masses were consistent with cellular angiofibroma. The testicular parenchyma and spermatic cord were grossly unremarkable. On microscopic examination of the testis, some atrophic seminiferous tubules were

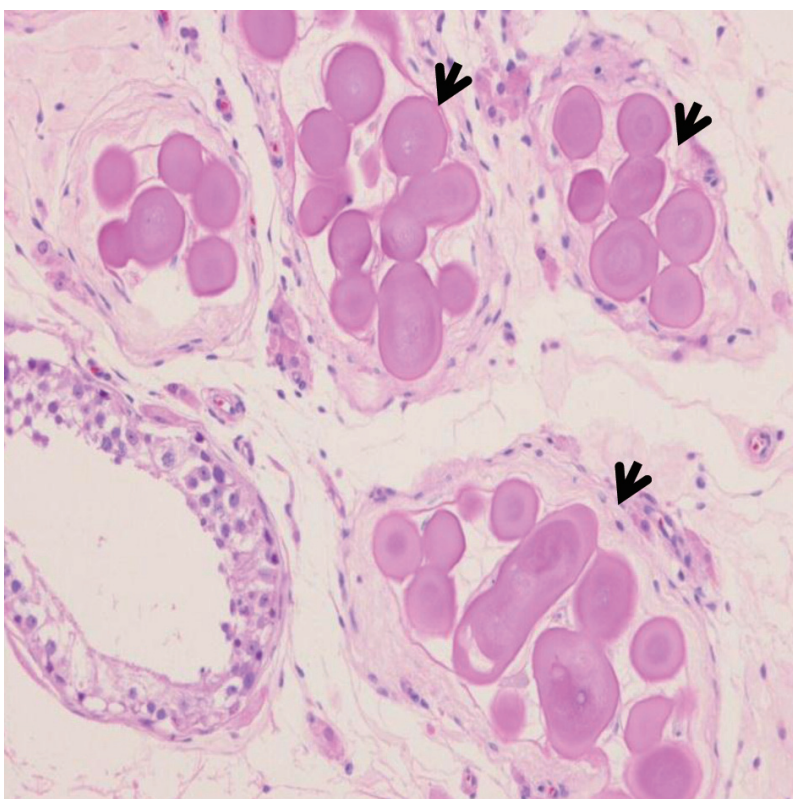

Fig. 3. Intratubular microcalcification. There are numerous completely hyalinized ghost tubules with corpus amylacea-like laminated intratubular bodies (arrows).

present and showed hyalinized degenerative change and contained corpora amylacea-like laminated eosinophilic bodies (Fig. 3). The remaining non-atrophic seminiferous tubules were unremarkable.

\section{DISCUSSION}

Cellular angiofibroma usually occurs as a solitary and circumscribed lesion in the inguinoscrotal region of men. ${ }^{1-5,7}$ Multiple cellular angiofibromas are very rare. To our knowledge, double cellular angiofibromas have not been reported. ${ }^{1-7}$ Although the pathogenesis of multiple cellular angiofibroma is unknown, we speculate that it might be associated with certain cytogenetic alterations. ${ }^{10}$ Cellular angiofibroma appears to be closely related to spindle cell lipoma and mammary-type myofibroblastoma, which show overlapping histologic and immunophenotypic features. ${ }^{4}$ Recent evidence has shown that these three tumors have similar common molecular genetic features, such as monoallelic deletion of RB1 and FOXO1, both of which reside within 13q14.,7 One of the interesting findings of the testicular parenchyma in this report was the presence of corpora amylacea-like bodies, suggesting laminated testicular microlithiasis. Testicular microlithiasis is relatively rare, and the exact incidence in normal and diseased testes is unknown. ${ }^{8,9}$ Laminated testicular microlithiasis has been associated with normal, cryptorchid testis, germ cell tumor, or atrophic seminiferous tubules. ${ }^{8}$ In this case, laminated 
testicular microlithiasis was associated with atrophic seminiferous tubules. Although the patient presented with self-limited hemospermia, a direct relationship between microcalcification and hemospermia could not be determined. The patient has been followed up, and no recurrence has been reported. In summary, we described a rare para-testicular double cellular angiofibroma with synchronous lesion of testicular microlithiasis. Although these two lesions might represent a coincidental finding, the relationship between these two lesions awaits further elucidation.

\section{Conflicts of Interest}

No potential conflict of interest relevant to this article was reported.

\section{REFERENCES}

1. Nucci MR, Granter SR, Fletcher CD. Cellular angiofibroma: a benign neoplasm distinct from angiomyofibroblastoma and spindle cell lipoma. Am J Surg Pathol 1997; 21: 636-44.

2. Iwasa $Y$, Fletcher CD. Cellular angiofibroma: clinicopathologic and immunohistochemical analysis of 51 cases. Am J Surg Pathol 2004; 28: $1426-35$.
3. Flucke U, van Krieken JH, Mentzel T. Cellular angiofibroma: analysis of 25 cases emphasizing its relationship to spindle cell lipoma and mammary-type myofibroblastoma. Mod Pathol 2011; 24: 82-9.

4. Chen E, Fletcher CD. Cellular angiofibroma with atypia or sarcomatous transformation: clinicopathologic analysis of 13 cases. Am J Surg Pathol 2010; 34: 707-14.

5. Sabah M, Mohan P, Kay E. Para-testicular cellular angiofibroma: a rare tumour in a male renal transplant patient. Virchows Arch 2006; 449: 489-92.

6. Choi HJ, Kim SN, Kim KR. Cellular angiofibroma of the vulva: a report of three cases. Korean J Pathol 2001; 35: 259-62.

7. Arakaki K, Chinen K, Kamiya M, et al. Evidence for an association between increased oxidative stress and derangement of FOXO1 signaling in tumorigenesis of a cellular angiofibroma with monoallelic 13q14: a case report. Int J Clin Exp Pathol 2014; 7: 8972-9.

8. Renshaw AA. Testicular calcifications: incidence, histology and proposed pathological criteria for testicular microlithiasis. J Urol 1998; 160: $1625-8$.

9. Zastrow S, Hakenberg OW, Wirth MP. Significance of testicular microlithiasis. Urol Int 2005; 75: 3-7.

10. Fanburg-Smith JC, Devaney KO, Miettinen M, Weiss SW. Multiple spindle cell lipomas: a report of 7 familial and 11 nonfamilial cases. Am J Surg Pathol 1998; 22: 40-8. 